\title{
DURATION LIMIT OF LASER PULSES EMITTED FROM A Ce-DOPED CRYSTAL SHORT CAVITY
}

\author{
Le Hoang Hai* \\ Le Quy Don University of Engineering, 100 Hoang Quoc Viet Rd., Hanoi, Vietnam
}

Nguyen Dai Hung

Institute of Physics and Electronics, 10 Dao Tan Str., Ba Dinh Dist., Hanoi, Vietnam

Hoang Huu Hoa

University of Hue, 2 Le Loi Str., Hue, Vietnam

Alex V. Quema, Nobuhiko Sarukura

Institute for Molecular Science, Myodaiji, Okazaki, Aichi 444-8585, Japan

Received 27 November 2006

\begin{abstract}
Based on the rate equation set for broadband cavities, the dependence of pulse duration on cavity and pumping parameters is analyzed. The cavity uses a Ce-doped crystal as a gain medium. Computation results show the variation of the pulse width with the change of cavity length, mirror reflectivity, pumping energy and pumping pulse duration. A significant influence of multiple-pulse operation in limiting pulse duration is realized and a pulse-width of the order $200 \mathrm{ps}$ is found to be the limit for the direct generation of ultraviolet single picosecond pulses from a Ce:LLF short cavity.
\end{abstract}

Keywords: short-pulse laser, solid-state laser, UV short-pulse, Ce-doped crystal

Ultrashort-pulse generation and amplification in the ultraviolet (UV) region have received a great deal of interest for numerous applications. However, it is difficult to determine which media are able to lase in the UV region. In the last decade, some solid-state tunable UV-laser materials such as Ce-doped media were found and have proven their vast potential in producing short and ultrashort UV pulses. There are a number of reports on the experimental use of Ce:doped media in generating ultrashort pulses [1 - 12]. However, all known UV ultrashortpulse master-oscillator-power-amplifier systems consist of mode-locked Ti:sapphire lasers [10 12] or various dye-laser-based oscillator stages and subsequent non-linear-frequency conversion systems or excimer power-amplifier stages. The above systems are extremely complicated and bulky, and such drawbacks restrict their applications. In view of these limitations, the direct acquisition of ultrashort seed pulses from a Ce-doped media oscillator is thus warranted. Due to

${ }^{*}$ Corresponding author e-mail: hai_hien_bi@yahoo.com 
the lack of $\mathrm{CW}$ pumping laser, low-Q and short cavity is a dominant technique for the generation of picosecond UV laser pulses from Ce doped media [2 - 6]. At present, the shortest UV pulse that has been generated directly from a cavity that uses the Ce-doped media as a gain medium is about $600 \mathrm{ps}[3,4]$. In this paper, the dependence of pulse duration on cavity and pumping parameters will be analyzed using the rate equation model. A significant influence of multiple-pulse operation in limiting pulse duration is realized and a pulse-width of the order 200 ps is found to be the limit for the direct generation of ultraviolet single picosecond pulses from a $\mathrm{Ce}^{3+}: \mathrm{LiLuF}_{4}(\mathrm{Ce}: \mathrm{LLF})$ short cavity.

The laser cavity constitutes of a cylindrical Ce-doped crystal rod bounded at both ends by mirrors with reflectivities of $R_{1}$ and $R_{2}$. This cavity is pumped transversally by another laser. The pumping beam is focused by a cylindrical lens into a line width of $\sim 0.1 \mathrm{~mm}$ on a side of the crystal rod. The model used for the Ce-doped media is the two-level system with populations $\mathrm{N}_{0}$ (lower level) and $\mathrm{N}_{1}$ (upper level). Assuming that the total concentration of Ce ion is constant, the population inversion and laser intensity can be described by the following rate equation system [13]:

$$
\begin{gathered}
\frac{\partial N_{1}}{\partial_{t}}=\left(\sigma_{a p} I_{p}+\sum_{i=1}^{n} \sigma_{a i} I_{i}\right) N_{0}-\left(\frac{1}{\tau}+\sum_{i=1}^{n} \sigma_{e i} I_{i}\right) N_{1} \\
\frac{\partial \boldsymbol{I}_{i}}{\partial t}=\left[2\left(\sigma_{e i} N_{1}-\sigma_{a i} N_{0}\right) l-\alpha_{i}\right] \frac{I_{i}}{T}+A_{i} N_{1}
\end{gathered}
$$

where $I_{\mathrm{i}}$ is the laser intensity at wavelength $\lambda_{\mathrm{i}} ; I_{p}$ is the pumping intensity; $\tau$ is the fluorescence lifetime; $\alpha_{\mathrm{i}}$ is the round-trip loss at $\lambda_{\mathrm{i}} ; A_{\mathrm{i}}$ is the constant that simulates the spontaneous emission; $\sigma_{\mathrm{ai}}$ and $\sigma_{\mathrm{ei}}$ are the absorption and stimulated emission cross sections at $\lambda_{\mathrm{i}}$, respectively; $\sigma_{\mathrm{ap}}$ is the absorption cross section at pumping wavelength; $T=2[L+l(n-1)] c^{-1}$ is the round-trip cavity time. Here, $l$ is the crystal length, $L$ is the cavity length, $n$ is the index of refraction and $c$ is the light velocity.

The above system of rate equations can be extended to an arbitrary number of wavelengths so that they cover all of the possible emission spectra. The shape of the pumping pulse is assumed to be a smooth Gaussian. The following results are obtained by solving the above rate equations for the cavity which uses Ce:LLF as gain medium (concentration of $3 \times 10^{17}$ ions $/ \mathrm{cm}^{3}$ ) and $\mathrm{KrF}$ laser (wavelength $248 \mathrm{~nm}$ ) as pumping sourse. The spectroscopic data for the medium mentioned are taken from Refs. [1, 2, 4, 9, 14].

It is well known that in pulsed laser operation, the span of the laser pulse is determined by the population inversion, which in turn, depends on the pumping level, spontaneous decay, stimulated emission, rate of build-up and decay of photons in the cavity due to the stimulated emission and various loss mechanisms. The accumulation of the excited-state well above threshold population is necessary for lasing. At the laser onset, the population inversion drops below threshold and the laser stops. After this, the population can again be built-up causing a sufficiently large inversion and thereby leading to the recommencement of laser action. Figure 1 shows the computation results of a laser emission from a $1.5 \mathrm{~cm}$ long cavity with a Ce:LLF crystal of the same length and using two mirrors with $70 \%$ and $5 \%$ reflectivities. The cavity is pumped by a pulse with a 10 ns duration (FWHM). Obviously, a single picosecond pulse (270 ps at FWHM) can be produced (Fig. 1a) at a pumping energy of $40 \mathrm{~mJ}$. As the pumping energy is increased, the cavity gradually enters multiple pulse operation in which the laser output takes the form of multi-pulse due to the spiking phenomenon (Fig. 1b). This result agrees with the experimental observation in Refs. [2, 4]. Thus, the generation of single picosecond pulses from Ce:LLF cavity is highly possible provided that the cavity and the pumping parameters are suitably selected. The duration of the first spike is determined by the time interval between the maximum and the first minimum points in the population curve. In other words, the pulse-width is determined by the rate of the drop in the population inversion during the laser onset and the 178 
span of such a drop are both affected by the cavity and the pumping parameters. In the rest of this paper, the discussion is confined to the single pulse operation of the cavity.
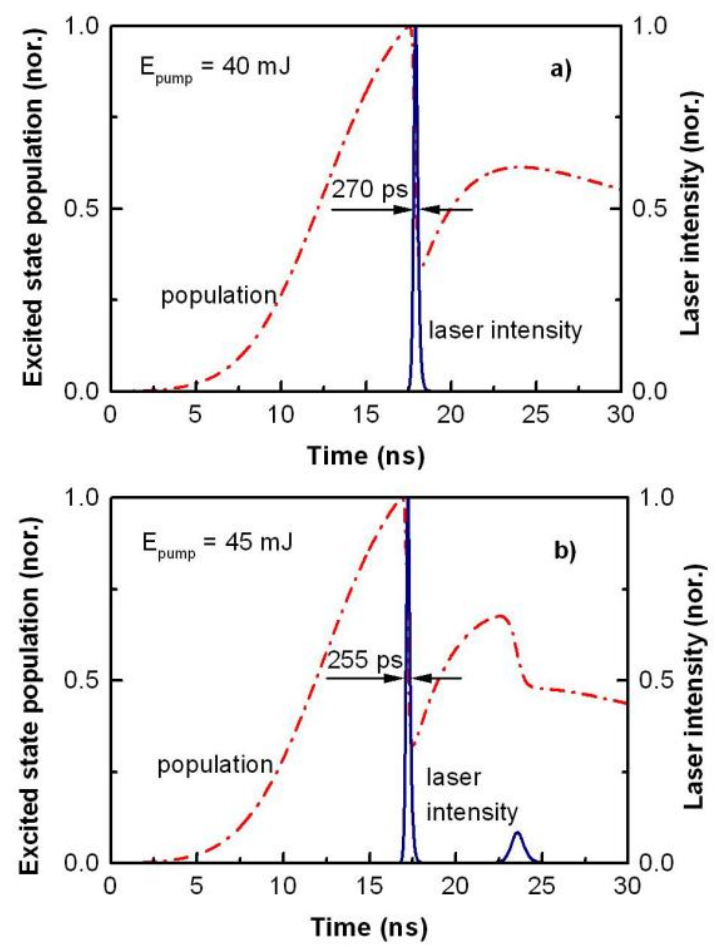

Fig. 1: Dynamics of laser emission from a cavity of $L=l=1.5 \mathrm{~cm}, R_{1}=70 \%, R_{2}=5 \%$ and pumped by pulses of $10 \mathrm{~ns}$ (FWHM) with pulse energy of (a) $40 \mathrm{~mJ}$ and (b) $45 \mathrm{~mJ}$. Dash-dot curves show the population of excited state while the solid curves signify the time profile of laser pulse

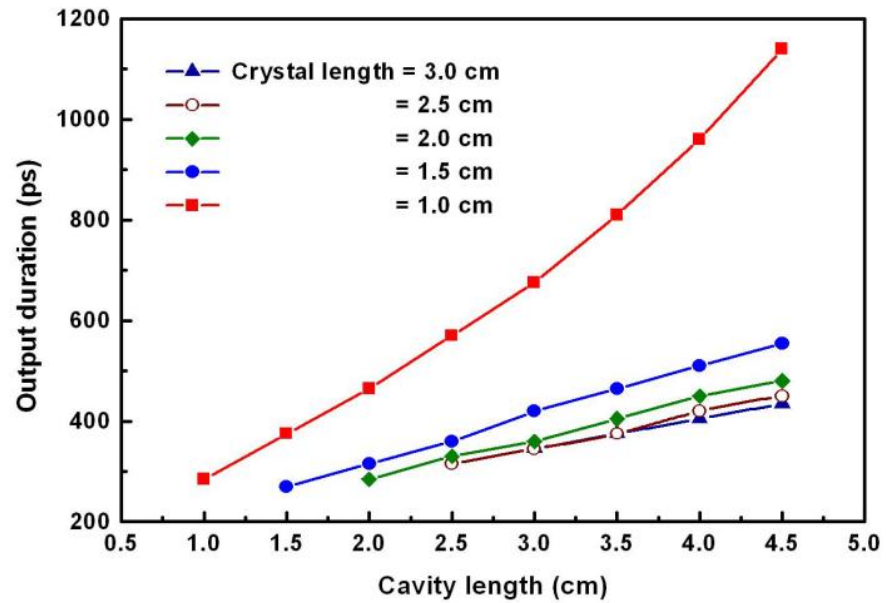

Fig. 2: Duration of UV laser pulse vs. cavity length. The cavity consists of two mirrors with reflectivities $70 \%$ and $5 \%$, and pumped by pulses of $10 \mathrm{~ns}$ and $40 \mathrm{~mJ}$ 
The variation of output pulse-width vs. cavity length is presented on Fig. 2. These results are obtained from a cavity with mirrors of 70 and 5\% reflectivities, and pumped by $10 \mathrm{~ns}$ (FWHM), $40 \mathrm{~mJ}$ pulses from a KrF laser. As seen in this figure, the output pulses become shorter and shorter with a decreasing cavity length. In addition, the rate of decrease in pulse duration is different at various crystal lengths and its slope increases with a shorter crystal length. The shortest pulse of $270 \mathrm{ps}$ can be obtained from a cavity of $1.5 \mathrm{~cm}$ long and a crystal of the same length. At a given cavity length, as the crystal length increases the output duration decreases.

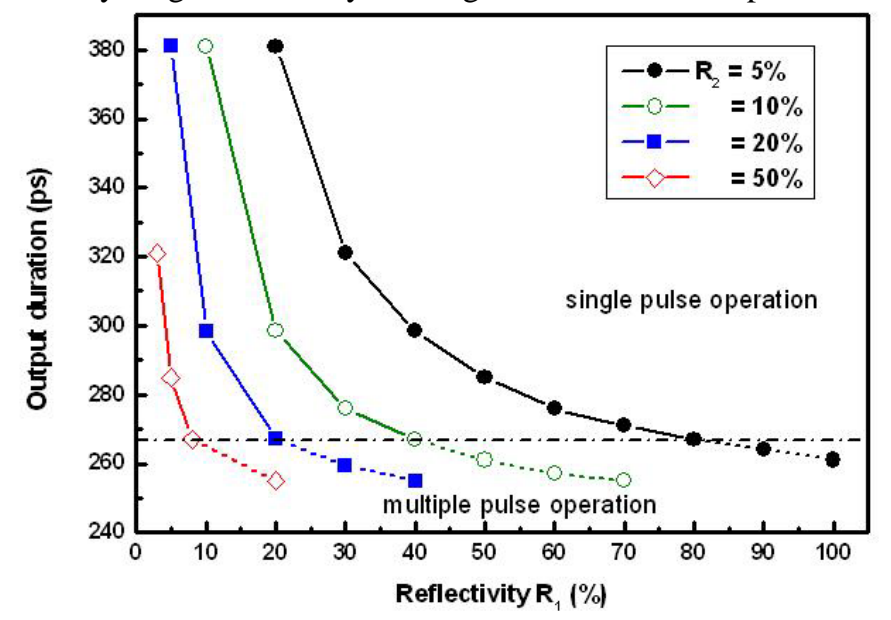

Fig. 3: Plot of output duration vs. mirror reflectivity. The cavity is $1.5 \mathrm{~cm}$ long with a crystal of the same length and pumped by $10 \mathrm{~ns}, 40 \mathrm{~mJ}$ pulses. The region above the dash-dot line corresponds to the single pulse operation while the region below the line denotes the multiple-pulse operation

Figure 3 shows the output duration variation vs. mirror reflectivity. This result is obtained with a cavity length $L=l=1.5 \mathrm{~cm}$ and a pumping energy of $40 \mathrm{~mJ}$. As seen here, with a given mirror of reflectivity $R_{1}$, the output duration reduces with increasing reflectivity $R_{2}$ of the other mirror. Strictly speaking, the output duration does not depend separately on either mirrors of the cavity. Then again, the output duration depends on both mirrors as a whole. Particularly, it depends on product of $R_{1} R_{2}$. This is typical because the product $R_{1} R_{2}$ is one of the factors that determine the $\mathrm{Q}$ parameter of the cavity. Accordingly, the output pulse-width decreases with increasing $\mathrm{Q}$ of the cavity. Obviously, the shortest possible pulse is always limited by the multiple pulse operation of the cavity. However, it should be noted here that this rule is only true in a limited region of reflectivity in which the single pulse operation of the cavity is sustained.

The above results can be understood by considering the difference between the maximum population inversion and the lasing threshold. With a given crystal length and pumping energy, a shorter cavity length and/or higher mirror reflectivity resulted in lower lasing threshold. Whereas, the maximum accumulated population of the upper laser level depends only on the crystal length and the pumping energy, which in this case are kept constant. Consequently, an increase in the difference between population inversion maximum and lasing threshold leads to higher drop rate in population inversion when the laser starts. As a result, the output pulse shortens with decreasing cavity length and/or increasing mirror reflectivity.

We did the computation for the Ce:LLF laser using the values $\mathrm{R}_{1}=70 \%, \mathrm{R}_{2}=5 \%, L=l=1.5$ $\mathrm{cm}$ with different durations of the pumping pulse. The results are shown in Fig. 4. As seen in this figure, with a given pumping energy, the output pulse-width decreases and eventually approaches to a constant value as the pumping pulse duration is decreased. Additionally, this constant value is dependent on the pumping energy since it goes to a lower value at higher pumping energies. Obviously, there exists an ultimate duration for the output pulse wherein 180 
much shorter output pulses would be unattainable by simply using pumping pulses of less than a few nanoseconds in duration. This numerical evaluation agrees rather well with the experimental observation in Ref. [9]. Since very short pulses are used for pumping, the cavity cannot follow the pumping intensity thereby creating a lag of output pulses with respect to the pumping pulse.

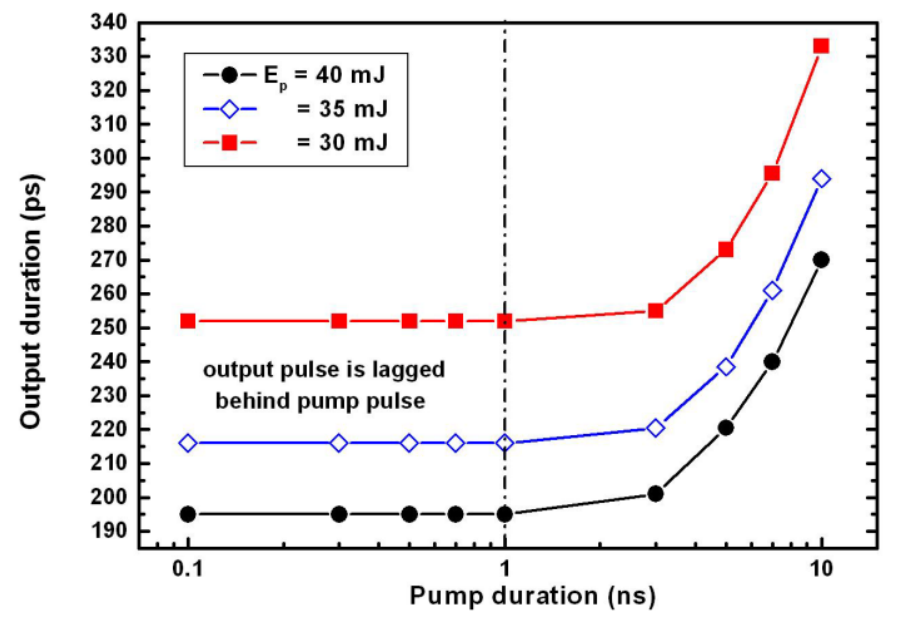

Fig. 4: Output duration vs. pump duration. The cavity is $1.5 \mathrm{~cm}$ long with a crystal of the same length, $R_{1}=70 \%, R_{2}=5 \%$. The ultimate duration is shorter with higher pumping energy. Ultimate durations 195 ps, 216 ps and 252 ps corresponds to pumping energies $40 \mathrm{~mJ}, 35 \mathrm{~mJ}$ and $30 \mathrm{~mJ}$, respectively

At the same pumping energy, as the pump pulse duration becomes shorter; the accumulation in the excited-state becomes faster and leads to a higher population $\mathrm{N}_{1}$ at the moment of laser onset. So, in the case of pumping by short pulse, a strong first spike can be obtained. After that, it is difficult to build up again the excited-state needed for the population inversion. However, if it is pumped by a sufficiently short pulse, the output pulse duration reaches the constant value due to the "saturation" of the dynamic response capability of the cavity.

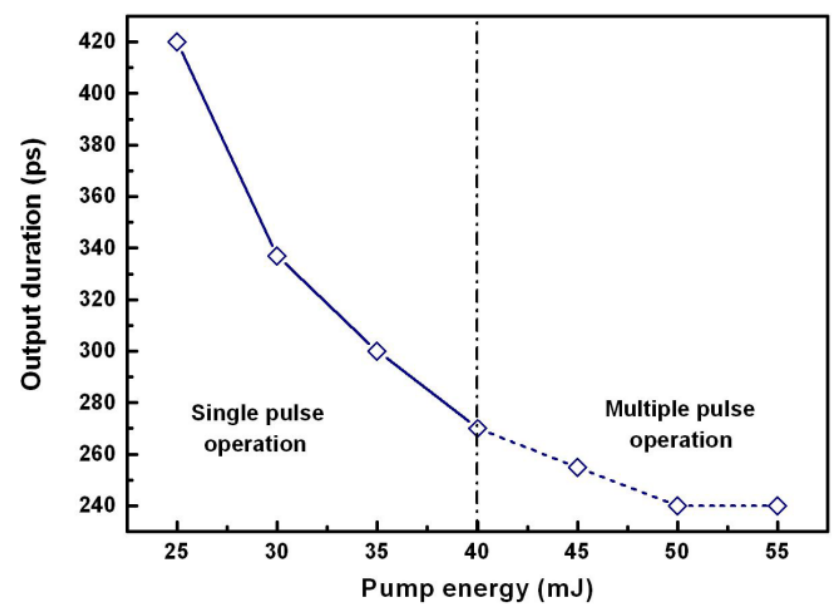

Fig. 5: Output duration vs. pumping energy at a constant pumping pulse-width of $10 \mathrm{~ns}$. The 1.5 $\mathrm{cm}$ long cavity contains a crystal rod of $1.5 \mathrm{~cm}$ long and bounded with mirrors of $70 \%$ and $5 \%$ in reflectivity 
As mentioned above, the pumping energy affects strongly the time profile of the output pulse. This effect is detailed in Fig. 5 using the computation results for a cavity of $L=l=1.5 \mathrm{~cm}, \mathrm{R}_{1}=$ $70 \%, \mathrm{R}_{2}=5 \%$ and various pumping energies. As we can see, the output pulse-width is decreased with the increasing pumping energy. Nevertheless, at high pumping levels, the cavity may work in the multiple-pulse regime. This result can be ascribed to the larger number of excited ions at a higher pumping energy and a repopulating capability of the excited state after the first emission.

Based on the above analyses, it seems that the use of a very short cavity with a appropriate pump energy and duration can generate single ultrashort pulses. But in that case, it is necessary to use a gain medium with a very high concentration of activated ions. This requirement is very difficult to satisfy and the feasibility of fabricating such gain medium is rather remote since it may lead to various problems in the crystal growth. Consequently, in the case of the Ce-doped crystal laser, the concentration of activated ions is a limiting factor of the pulse duration.

In conclusion, the analyses detailed here demonstrate that UV single picosecond laser pulses can be directly generated from a Ce:LLF short cavity. Due to their influences, a suitable selection of cavity and pumping parameters is a critical task that will determine how short a pulse can be generated. In order to attain the shortest possible single picosecond pulses, cavity and pumping parameters should be carefully selected so that multiple pulse operation would be eliminated. The computation results obtained here can provide an effective guide principle for scaling Ce:doped UV short-pulse laser oscillators. In addition, as demonstrated earlier by the numerical evaluations, the duration of output single pulses from a Ce:LLF short cavity is limited at around 200 ps.

\section{REFERENCES}

1. Sarukura, N., Liu, Z., Segawa, Y., Edamatsu, K., Suzuki, Y., Itoh, T., Semashko, V.V., Naumov, A.K., Korableva, S.L., Abdulsabirov, R.Yu., and Dubinskii, M.A. (1995), Opt. Lett., vol. 20, pp. 294-296.

2. Sarukura, N., Liu, Z., Segawa, Y., Semashko, V.V., Naumov, A.K., Korableva, S.L., Abdulsabirov, R.Yu., and Dubinskii, M.A. (1995), Opt. Lett., vol. 20, pp. 599-601.

3. Sarukura, N., Liu, Z., Segawa, Y., Semashko, V.V., Naumov, A.K., Korableva, S.L., Abdulsabirov, R.Yu., and Dubinskii, M.A. (1995), Appl. Phys. Lett., vol. 67, pp. 602-604.

4. Sarukura, N., Dubinskii, M.A., Liu, Z., Semashko, V.V., Naumov, A.K., Korableva, S.L., Abdulsabirov, R.Yu., Edamatsu, K., Suzuki, Y., Itoh, T., and Segawa, Y. (1995), IEEE J. Quantum Electron., vol. 1, pp. 792-804.

5. Sarukura, N., Liu, Z., Ohtake, H., Segawa, Y., Dubinskii, M.A., Semashko, V.V., Naumov, A.K., Korableva, S.L., and Abdulsabirov, R.Yu. (1997), Opt. Lett., vol. 22, pp. 994-996.

6. Liu, Z., Ohtake, H., Sarukura, N., Dubinskii, M.A., Semashko, V.V., Naumov, A.K., Korableva, S.L., and Abdulsabirov, R.Yu. (1997), Jpn. J. Appl. Phys., vol. 36, pp. L1384L1386.

7. Sarukura, N., Liu, Z., Izumida, S., Dubinskii, M.A., Abdulsabirov, R.Yu., and Korableva, S.L. (1998), Appl. Opt., vol. 37, pp. 6446-6448.

8. Liu, Z., Sarukura, N., Dubinskii, M.A., Semashko, V.V., Naumov, A.K., Korableva, S.L., and Abdulsabirov, R.Yu. (1998), Jpn. J. Appl. Phys., vol. 37, pp. L36-L38.

9. Liu, Z., Sarukura, N., Dubinskii, M.A., Abdulsabirov, R.Yu., and Korableva, S.L. (1999), J. Nonlinear Opt. Phys. Materials, vol. 8, pp. 41-54. 
10. Liu, Z., Kozeki, T., Suzuki, Y., Sarukura, N., Shimamura, K., Fukuda, T., Hirano, M., and Hosono, H. (2001), Jpn. J. Appl. Phys., vol. 40, pp. 2308-2309.

11. Liu, Z., Kozeki, T., Suzuki, Y., Sarukura, N., Shimamura, K., Fukuda, T., Hirano, M., and Hosono, H. (2001), Opt. Lett., vol. 26, pp. 301-303.

12. Liu, Z., Kozeki, T., Suzuki, Y., Sarukura, N., Shimamura, K., Fukuda, T., Hirano, M., and Hosono, H. (2001), IEEE J. Quantum Electron., vol. 7., pp. 1-9.

13. Hung, N.D., Hoa, H.H., Hai, L.H., Brechignac, P. (1999), Appl. Phys., vol. B 69, pp. 467471.

14. Marshall, C.D., Peth, A., Payne, S.A., Krupke, W.F., Quarles, G.J., Castillo, V., Chai, B.H.T. (1994), J. Opt. Soc. Am., vol. B 11, pp. 2054-2065. 